\title{
Context-Aware Media Recommendations
}

\author{
Abayomi Moradeyo Otebolaku \\ Telecommunications and Multimedia Unit, INESC TEC \\ Faculty of Engineering, University of Porto, Portugal \\ abayomi.otebolaku@inescporto.pt
}

\author{
Maria Teresa Andrade \\ Telecommunications and Multimedia Unit, INESC TEC \\ Faculty of Engineering, University of Porto \\ Porto, Portugal \\ mandrade@fe.up.pt
}

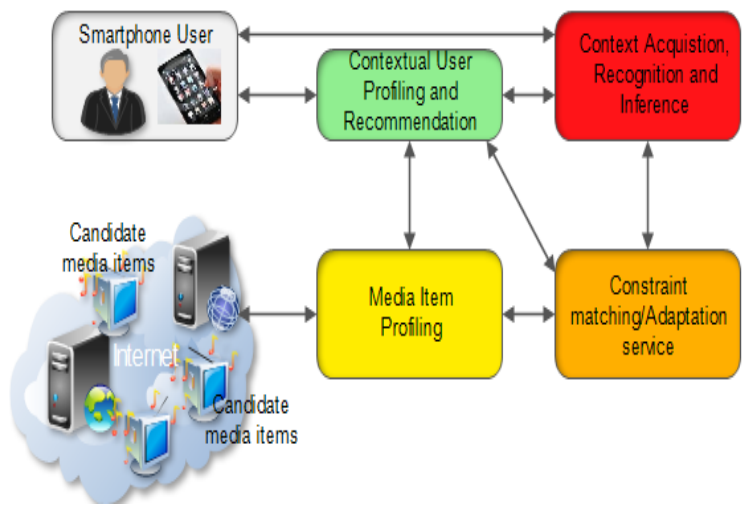

Abstract - Media content recommendations for a mobile user based on his changing contextual preferences, otherwise called context-aware media recommendations, constitute a very important challenge. Context-aware media recommendation systems take context information such as user preferences, activities, time, location, device, and network capabilities as inputs for media recommendations, whereas the traditional recommendation systems use only user preferences in the form of ratings to deliver media recommendations. This paper presents a generic high-level architecture of context-aware recommendations, discussing its key techniques and solutions, which are based on context acquisition, recognition, and representations, using MPEG-21 and ontology model, and a contextual user profiling process, as well as MPEG-7 for media description model and media presentation adaptation.

Keywords—context awareness; contextual user profile; contextaware recommendation; mobile phones; media items

\section{INTRODUCTION}

Because of the rapid development of mobile and Internet technologies, in addition to the unprecedented breakthrough in wireless communication technologies, massive and diverse online-based media contents are now available. These trends have afforded mobile users ubiquitous access to online-based rich media items, using various types of smart devices such as smartphones, PDA, and tablets. However, with this development, obtaining relevant media items remains problematic for mobile users [1-3]. For example, existing solutions do not take into account the changes in mobile user's preferences with location and other contextual information [4, $7,9]$, they also do not consider that smart devices have varying capabilities, and that the wireless networks are heterogeneous. Therefore, recommendation techniques in the traditional environment cannot work in a mobile environment, because these technical difficulties can prevent effective and seamless delivery of relevant online-based rich media items to mobile users.

Consequently, recommendation systems in mobile environments should deliver contextually relevant media content to the appropriate user according to his current contextual situation. Context-aware media recommendation is an important solution that can address the above mentioned challenges by delivering and customizing rich media content based on user's changing contextual preferences. Unlike the traditional recommendation systems, context-aware media recommendation systems can provide effective solutions to the problems faced in mobile computing environments by mobile users when consuming online based media items. To realize these solutions, context-aware recommendation can provide relevant items, at the same time adapting these contents to the user's device and wireless network capabilities.
Fig. 1. The proposed system's high-level architecture

Consider this archetypal scenario, for instance. Sitting at home on a Friday at 8:30 PM, Ana enjoys watching video clips of recent movies on her smartphone. She relies on the media recommendation service to provide with her favorite recommendations, especially the ones her close friends (who are also media recommendation service subscribers) have preferred. She always wants to select one or two movies to watch on Saturday with her friends at the cinema, and she prefers to have such suggestions when she engages in some less demanding activity. However, her device battery runs down while previewing the recommended items.

In this scenario, with the existing solutions, it is not difficult to determine those movies that are relevant to Ana, based on her previous consumptions (if any) or those consumed by her friends. Nevertheless, if Ana prefers watching a romance movie when she is only with her partner, and the system suggests to her a movie called Insatiable, a romance movie, which her friends have previewed and liked, this would be a bad recommendation because Ana would like to watch the movie while she is with her partner at home. This type of mistake or preference conflicts can be avoided if the recommendation service is aware of Ana's contextual choices at the time of the recommendation. The existing systems lack the features, which can take care of the requirements such as those in the above scenario for implicit and explicit recommendations of rich media content to mobile users, without conflict of preferences. Fig. 1 shows the high-level view of the solution proposed to address these issues.

The purpose of this paper is to introduce a generic architecture for context-aware media recommendations, which we have proposed, presenting its key components and the techniques that can be used to realize them.

\section{RELATED WORK}

Most popular works in context-aware recommendations such as Pessemier et. al. [14], Chen [5], and Yu et.al.[3], focus on 
explicitly using context information to suggest media items. Cinemappy [13] uses the location and movie consumption history of mobile users to recommend relevant movies. [12] uses activities such as walking, running, studying, etc. to recommend music to mobile users, its weakness is that it considers only user activity and not location information or other context information that influences user preferences. Generally, the works above can only handle domain specific media items such as movie, music, news, etc. unlike our proposed solution, a generic architecture, which integrates a hybrid and generic system that incorporates a context model that can handle broader contextual information than just location information, as well as having the capability to accommodate more than one media content domain. In addition to user location, our work integrates user, including his device, network and environment information, and provides both explicit and implicit recommendation services to deliver relevant media items. The services provided by the architecture such as recommendation processes, contextual user profiling service, context management service, etc. are implementation platform neutral because they are designed as interfaces, which are exposed as web services, providing access from any mobile software and hardware platforms. It also includes an optional adaptation service that can tailor the presentations of the recommended contents to the device and network constraints.

\section{SYSTEM OVERVIEW}

Fig. 2 shows the ten collaborating components of the proposed context-aware media recommendation system. Media item profiling is responsible for filtering and extracting important media item metadata, which it crawls from the Web and stores in the media metadata repository. Context monitoring, acquisition, recognition, reasoning, learning, and inferencing are handled by the context manager, which aggregate low-level context information from the smart device's embedded sensors and infers high-level contextual information. The context inference contains a context knowledge base where important inferred context information is stored. The media recommendation service queries the context inference manager, contextual user profiling manager, and the media profiling manager for contextual information, contextual user preferences, and candidate media item descriptions respectively. The contextual user profile manager is responsible for determining the contextual preference for consumption in specific contextual situations and for learning the user's consumptions and interactions using machine learning techniques (such as Case Base Reasoning). The media recommendation manager implements services that recommend media items to the user. It determines the appropriate media category (such as news, music, movies, etc.) and invokes one of the recommendation services to predict the specific media

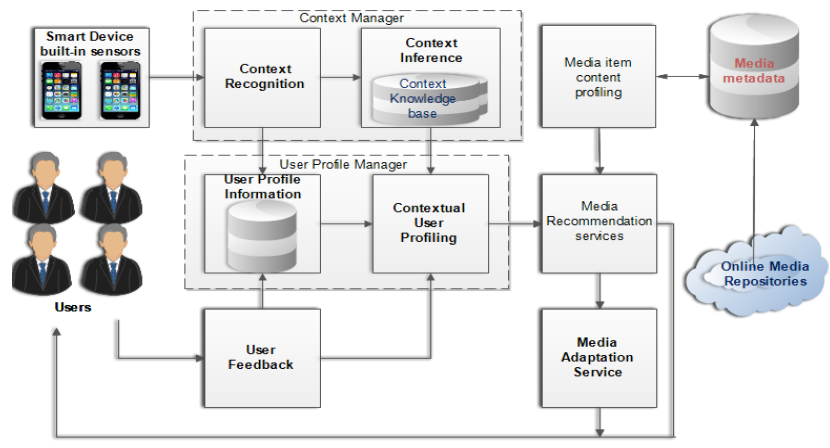

Fig. 2. Proposed system architecture

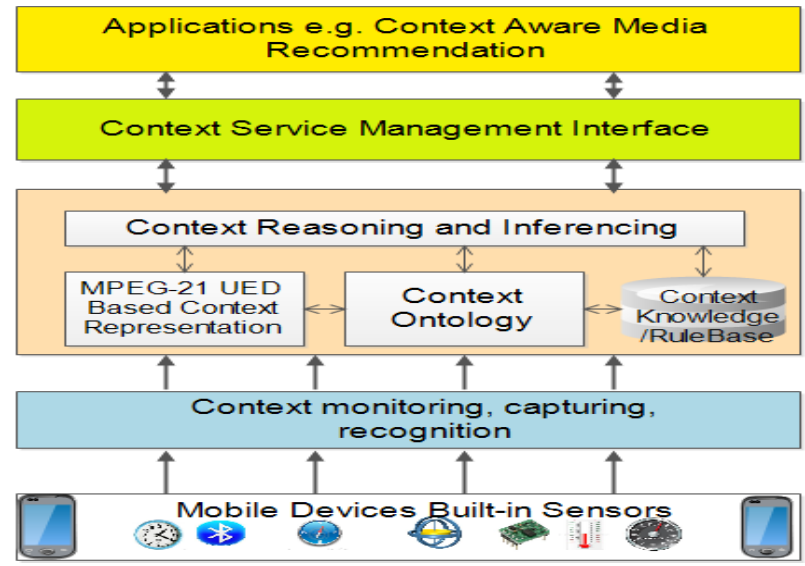

Fig. 3. Context management abstractions

item the user would prefer in that specific context. For instance, if it determines that it is the news item that matches the user's present preference and context, then it invokes either content based recommendation service or a hybrid recommendation service. It also determines if the variation of the media item being suggested is in the appropriate format for the user's device characteristics and present network condition, and then invokes the media presentation. Otherwise, it invokes the media adaptation service to adjust the format of the recommended media item accordingly.

\section{SYSTEM ARCHITECTURE}

\section{A. Media Content Profiling Management}

For efficient and interoperable processing of the media items, by the media recommendation and adaptation processes, the media metadata is represented in a standardized format, based on MPEG-7 description tools such as a Creation DS in addition to the Classification DS to describe the media item metadata such as the title of the item, its genre or other highlevel properties. MPEG-7 is an ISO/IEC standard developed by the Moving Picture Expert Group, MPEG. Formally, MPEG-7 "Multimedia Content Description Interface", is designed to describe multimedia contents [5].

To enable the adaptation of the recommended item's presentation, MPEG-7 Variation DS is adopted to specify the variations of the media items' contents to meet the specific capabilities of the network conditions as well as those of the user's smart devices. However, because online based media items are generally not described based on MPEG-7 description tools, the media profiling management provides alternative techniques based on web services to obtain media item metadata.

\section{B. Context and Activity Management}

The context manager, shown in Fig. 3, is responsible for a number of services, which are context monitoring, context acquisition, context recognition, context learning, context reasoning, and context inference. The context monitoring observes changes occurring in the smart device embedded sensor raw data, which are captured in the context acquisition process. The context recognition process obtains the captured sensor data, and performs a number of operations such as raw data preprocessing to eliminate data inconsistency and error, it extracts important features that are used to match the patterns of labeled context information, and then uses classification algorithm to identify the labeled high-level contexts corresponding to the extracted features $[8,15,22]$. 
The recognized context information is passed to the context learning and reasoning processes to infer a more meaningful context information by reasoning on one or more atomic context information, relating them to each other to obtain a real world contextual situation. To realize these processes, machine learning techniques can be implemented for classifying events emitted by the user's device embedded sensors and for recognizing high-level context information. The recognized context is represented and described using MPEG-21 user environment description tools (UEDs) [6]. To infer further high-level semantic context information from the basic recognized context information and to maintain context knowledge base consistency, we proposed a context Ontology model [20]. The OWL based context ontology is backed up with a semantic web rule language (SWRL) to specify different kinds of rules to support reasoning tasks of the context management service.

\section{Contextual User Profiling Service (CUPS)}

A user profile describes preferences of a user in the form of a summary, normally based on the history of the user's actions $[4,7]$. In our solution, it summarizes user's consumptions as a limited set of categories. Categories are described by a genre, in turn described by a number of properties. One or more genres may be associated with one category. Several properties may be associated with one genre. It incorporates the contextual dimension, associating one or more inferred context to each category-genre-property. Accordingly, it is possible to generate a contextual user profile for each inferred context, thus obtaining the preferences of the user in each specific context.

The contextual user profile service is responsible for the management of user preferences, which are based on the contextual situations of the users. As the user preferences change with time and contexts, to track these preference changes, we introduced lifetime parameter, which provides an indication of the time elapsed since the last preferred consumption occurred. The intensity measures the degree of importance or relevance the user attaches to the preferences.

CUPS works in tandem with the recommendation services to provide mobile users with relevant media items that dynamically satisfies his preferences, by matching the present and past contexts, a crucial process, which must be accurately executed to obtain the user's dynamic contextual preferences.

To realize this, a case base reasoning approach for retrieving and for learning the contextual user preferences based on the currently identified user context situation and past contextual situations and preferences is proposed. Case based reasoning (CBR) is a machine learning method that solves a new problem by remembering a previous similar situation, reusing information and knowledge in the situation [10]. It relies on the general knowledge of the problem domain or associating generalized relationships between problem description and conclusion [10]. It utilizes the specific knowledge of previously experienced, concrete problem situation (cases) to solve new problems. One unique and important feature of CBR is its ability for incremental and sustained learning from a new experience, which is retained each time a problem is solved, making such experience immediately available for future problems [10], [19]. The CBR compares the present and past contextual situations (with associated preferences). In CUPS, this is similar to solving a new contextual situation problem case by using past context based on the preferences of

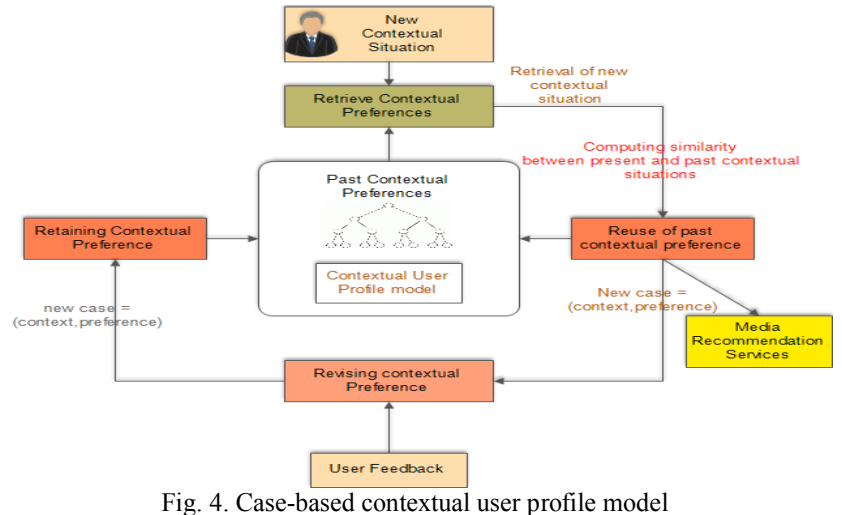

the users. Previously identified contextual situations and associated preferences constitute the past case, whereas the new case is the newly identified contextual situation whose corresponding preference is being determined. Therefore, a contextual preference case is described by a tuple <premise, value $>$. The premise describes a contextual situation and its characteristics, while the value is the contextual preference obtained based on the premise. Specifically, the premise is a contextual situation (C) of a mobile user when using his smart devices, whereas the value part is the contextual user preference in that situation, which is used in the recommendation process. A contextual situation (a case) is represented as $\mathrm{Cs}=\left\{\left(\mathrm{C}_{\mathrm{i}}, \operatorname{Pr}_{\mathrm{i}}\right)\right\}$. One of the preferences corresponding to the newly identified contextual situation is the value to be determined. To execute its operation, the proposed case based contextual user profile performs four operations as shown in Fig. 4.

1) Identifying the present contextual situation of the mobile user, which is obtained from the context management service.

2) Retrieving the most similar contextual situations and their respective contextual preferences.

3) Among the retrieved contextual preferences, reusing the ones most similar to the present contextual situation.

4) Revising, updating, and retaining the identified contextual situation and its corresponding context preference

a) If no matching contextual preference is found, add the new contextual situation and learn the user's interaction

b) If a match is found, add the new contextual situation and the corresponding contextual preference

The case based contextual user preference model is updated using user's explicit and implicit feedbacks to learn the contextual preferences for the recommended media items, which are related to the present contextual situation.

\section{Media Item Recommendation Service (MIRS)}

The media item recommendation service (MIRS) explores three recommendation algorithms for contextual media item recommendations. The content base $(\mathrm{CBF})$, the collaborative based (CF) and hybrid based recommendation algorithms [4]. In this article, we will not go into the details of each of these algorithms, but will only summarize their operations and how they are used in our proposed architecture.

The traditional collaborative recommendation generates recommendations for the target user based on the item previously co-rated or viewed by other users, and the contentbased approach that uses the consumption history of the user 


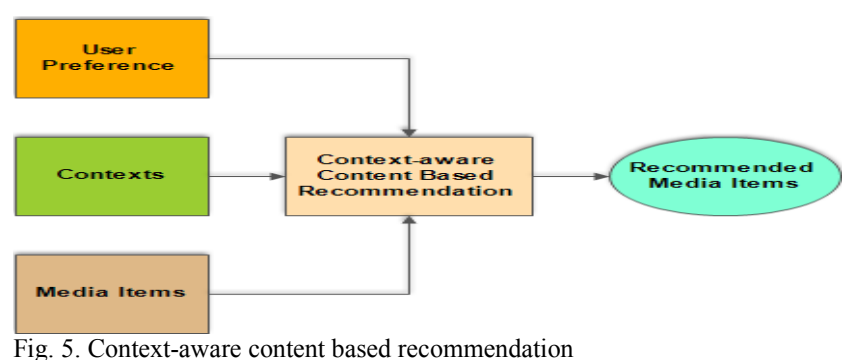

[21. These two approaches suffer from the so-called new user problem, which excludes casual users or those items the system does not have enough information about to generate recommendations [4]. Hybrid recommenders combine one or more of the conventional recommendation processes to overcome their individual weaknesses to gain better performance.

1) Context-aware content based recommendation

Because the traditional content based process suggests content to users based on their consumption history and descriptions of the available candidate content, it suffers from the overspecialization problem, where the system can only recommend media items that are similar to the one user has consumed in the past [4]. To address this limitation, we propose an extension of this process, as shown in Fig. 5, in conjunction with the contextual user profile model by suggesting multimedia items to users based on the contexts in which they have consumed content in the past and the descriptions of those consumed contents.

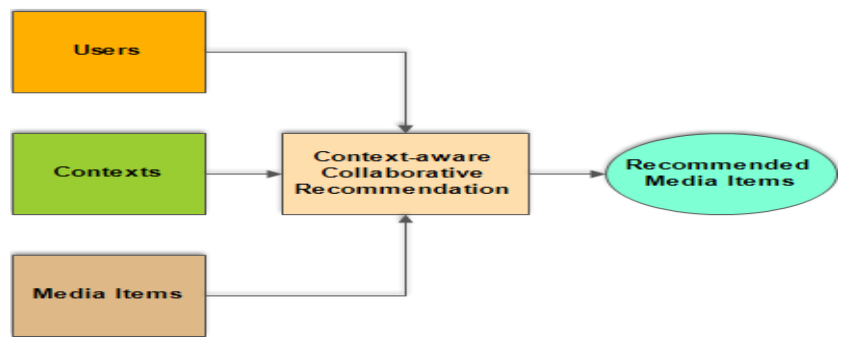

Fig. 6. Context-aware collaborative recommendation

\section{2) Context-aware collaborative recommendation}

Conventional collaborative recommendation suggests content to a target user based on the opinions and preferences of other users. This approach suffers from new user and new item problems resulting in sparse matrix [21]. It means that the recommendation system cannot effectively recommend suitable media items to a user who doesn't have adequate information in the system. In addition, it cannot recommend new media items, which have not been rated by a sufficient number of users. To address these problems, this process has been extended, as shown in Fig. 6, to suggest media items to the target user using the contexts in which active users who are similar to the target user have consumed the contents. The similarity between each user profile and the target user profile is calculated using Pearson correlation formula.

\section{3) Context-aware hybrid recommendation}

The traditional collaborative recommendation generates predictions for the target user based on the item previously rated or viewed by other users, and the content-based approach uses the consumption history of the user. These two approaches suffer from the so-called new user problem, which excludes casual users from recommendation. Any hybrid

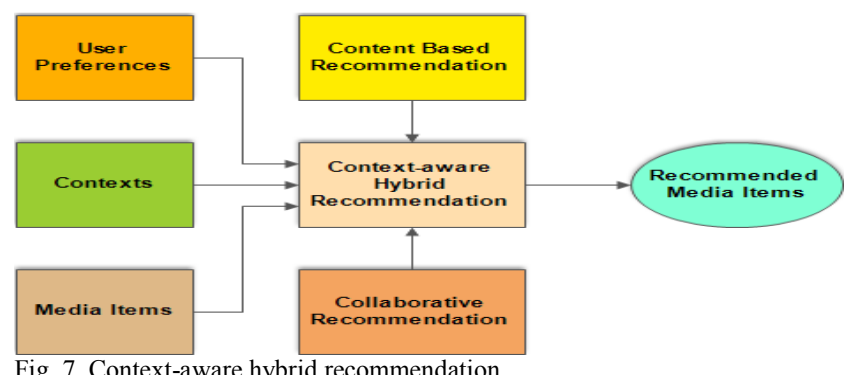

Fig. 7. Context-aware hybrid recommendation

Recommendation, using a combination of these methods also inherits the new user problem.

The context-aware hybrid recommender, shown in Fig. 7, combines conventional content based and collaborative based recommendation processes to overcome their individual weaknesses to gain better performance. To take advantage of the hybrid technique, we also propose a context-aware hybrid technique that combines the context-aware $\mathrm{CF}$ and $\mathrm{CBF}$. Basically, the hybrid model uses the contexts in which other users have consumed the content previously, to find users that are similar to the target user by comparing the active user's context history and the target user's present context. This is achieved in three phases. In the first phase, it identifies every user (neighbor) that is similar to the target user by searching through their profile trees, looking for contexts that match the target user's recognized context. For every user profile with a match, the intensity value of the category-genre-property nodes in the user profiles are retrieved. This value is then used to calculate the similarities between all users. After this calculation, it then selects the top $n$ most similar users, called neighbors or friends of the target user who have consumed contents in the same or similar contexts to the target user's current context.

In the second phase, it ranks the candidate media items for each neighbor by obtaining vectors $\mathrm{V}_{\mathrm{C}}$ and $\mathrm{V}_{\mathrm{M}}$ corresponding to contextual user profile of every neighbor and candidate media profile vectors respectively. It then applies the cosine formula to calculate the distance between these vectors.

In the third phase, it generates the predicted preference value for each candidate media item from the second phase for the target user, using Resnick [17] prediction. The Resnick prediction discounts the contribution of every neighbor's prediction according to its degree of similarity with the target user so that more neighbors have a large impact on the final intensity predictions [17]

Finally, recommendation list is built by ordering the candidate media items in descending order of magnitude of the computed prediction values.

MIRS performs the recommendation process in the following specific steps.

1) It initiates communications with the contextual user profile service to obtain the user's present contextual situation and corresponding preferences. The CUPS then communicates with the context service manager to obtain the user present contextual situation which CUPS uses to process the user profile to obtain the user contextual preferences and sends it to the MIRS.

2) MIRS determines the specific category of media items (e.g. news, movies, music, etc.) and uses this information to invoke the appropriate recommendation service. It implements three contextual recommendation services. Context-aware content based recommendation service, context-aware collaborative 


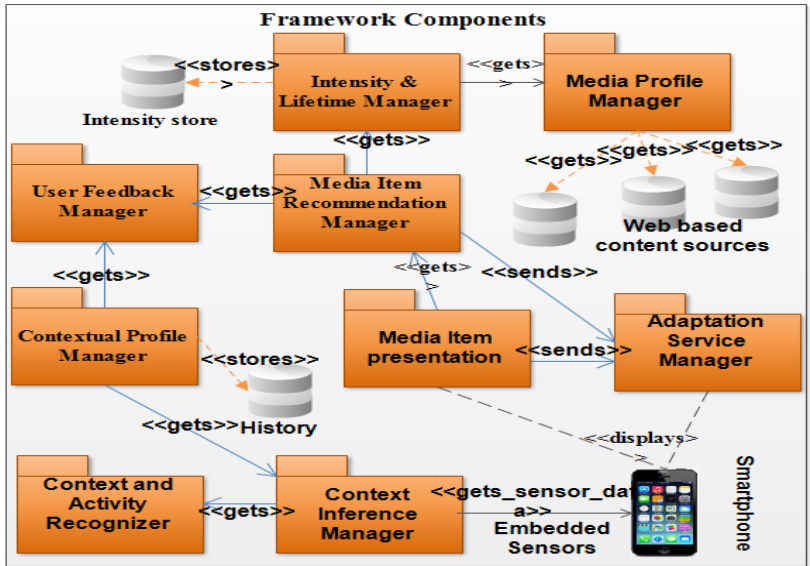

Fig. 8. Context-aware media recommendation component model

recommendation service and context-aware hybrid recommendation service.

4) After the appropriate recommendation process has been performed and a list of n-best media items is generated, the MIRS then evaluates the selected recommendation media item and the user's device capability and network conditions, adopting a SWRL rule based technique. The recommended media item's modality, frame rate, format, etc. has to satisfy the device constraints as well as the network conditions, namely screen size, media format support, network bandwidth, etc.

5) If the recommended media item's characteristics match the device and network constraints, then MIRS invokes the media item presentation to deliver the recommended media items to the user.

6) If the recommended media item characteristics do not match the device and network constraints, then MIRS invokes the media item adaptation service to adjust the or format, modality, etc. of the recommended items to suit the device and network constraints.

\section{E. Media Item Presentation Adaptation}

The media item presentation adaptation service is responsible for adapting media item contents based on summarization and transcoding techniques. The summarization summarizes content such as audio or video into short ones, according to the battery power remaining, network bandwidth functions, whereas media transcoding transforms the content from one media type to another based on the network conditions such as bandwidth and according to the device capabilities such as screen size or according to the type of media it is capable of playing [6]. The media item presentation adaptation service proposes to integrate ContextAware Ontologies for Multimedia Applications (MULTICAO) to provide adaptation services [23]. MULTICAO was developed using OWL DL with adequate provision for adaptation decisions relying on various rules based reasoning mechanisms that support the automatic inference over adaptation decision knowledge base. MULTICAO provides ADAPTATION Decision Engine (ADE), which is responsible for adaptation reasoning and decision-taking services, and a set of Adaptation Engine operation services, responsible for carrying out adaptation of media content, such as modification of the content into different modalities such as text, video, or images depending on the device and network constraints [11].

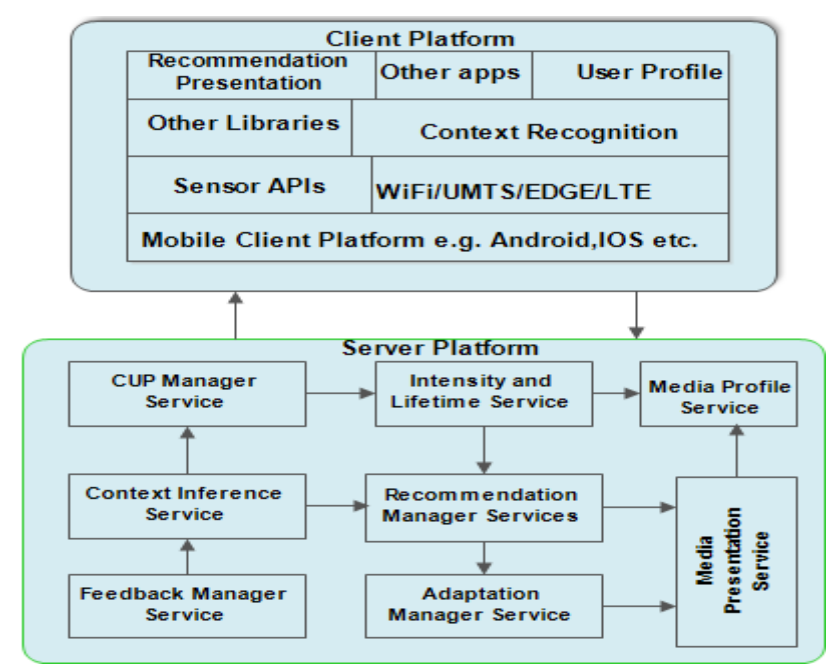

Fig.9. Implementation architecture of the context-aware recommendation application

\section{IMPLEMENTATION}

The implementation of the proposed context-aware media recommendation, based on its design, is platform neutral, i.e. it can be implemented on any platform. Fig. 8 is the component model of the architecture, representing each of the important services provided by the architecture. Basically, it can be implemented as a client-server system. The client services run on mobile devices, such as mobile phones. Parts of the context management service can be implemented on the smart device. For example, the context monitoring, capturing, and recognition processes can run on Android, IOS based devices, etc.

In the proposed system, the client services have been implemented on Android devices, whereas other components have been implemented based on Java EE 7 platform technologies, namely, the Enterprise Java Beans (EJB) for components, and Java Persistent API(JPA) to manage the interactions between the components and the databases.

Additionally, each of the components has been implemented as REST web services, and exposing them via public interfaces [18]. We have also demonstrated the feasibility of the system by developing mobile context-aware movie recommendation, which uses the services provided by the framework to recommend online-based movies to mobile users. The client-server implementation architecture is depicted in Fig. 9. Screenshots of the application are shown in Fig. 10, which presents the contextual profile management and context management visualizations, where a user can optionally manage her profile, and where his contextual information can be monitored. In the present application, three categories of content (movies, music, and news) are presently supported.

Fig. 11 presents the recommendation visualizations of the context-aware mobile recommendation application, showing the recommended movie content and all options available to user to visualize the recommendation, such as playing the movie trailers, or connecting to online sources for additional information on the recommended items. By selecting any of the recommended items in the list presented to the user, the application records and sends information about the selected items to the server. The server uses this information to implicitly update the user's contextual profile. 


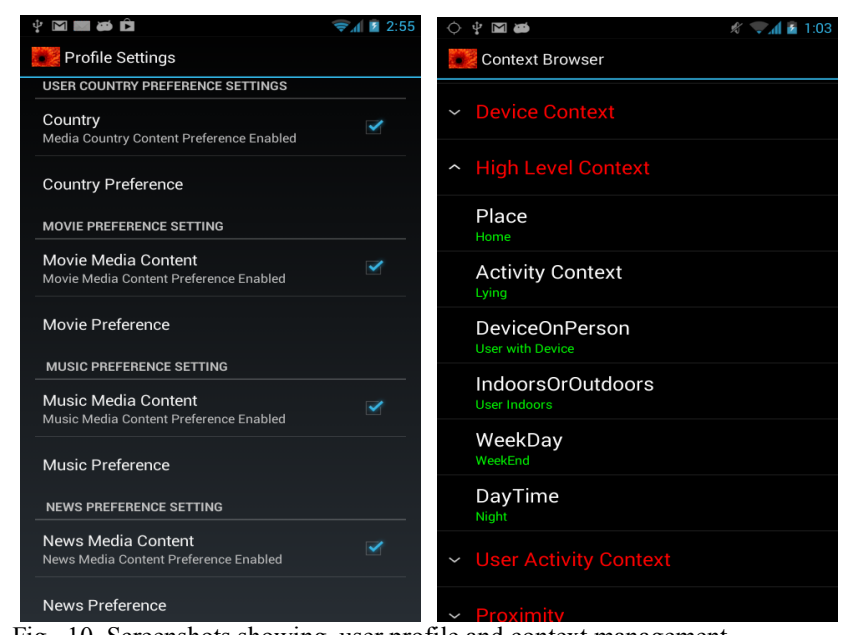

Fig. 10. Screenshots showing user profile and context management
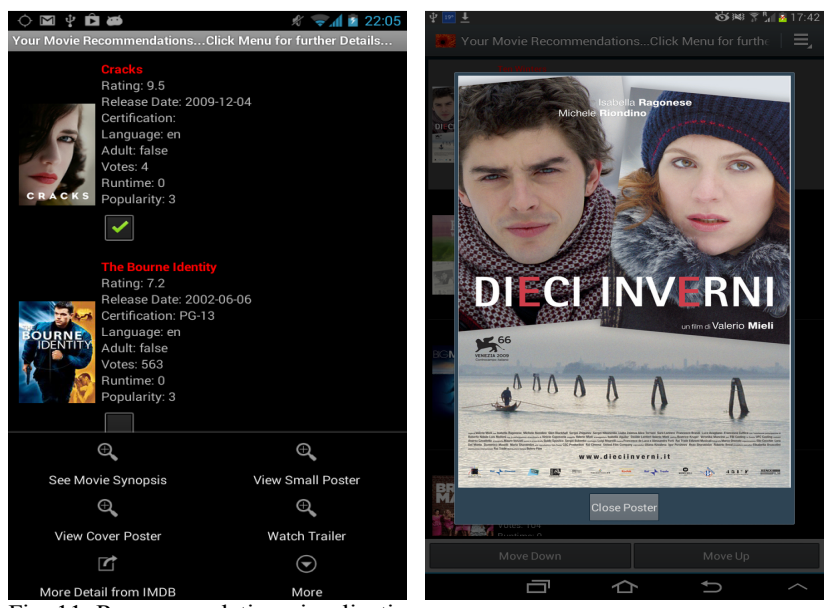

Fig. 11. Recommendation visualizations

\section{CONCLUSION AND FUTURE WORK}

This paper identifies and presents a conceptual and generic architecture that supports context-aware media recommendations, discussing its basic components, and its enabling technologies. The developed architecture is generic enough to handle various types of online-based media contents and its feasibility as been demonstrated via a context-aware mobile movie recommendation application.

In the future, we plan to apply the context-aware media recommendations to provide adaptable and contextually relevant media items such as music, news, and other onlinebased rich media items.

\section{ACKNOWLEDGMENT}

The work presented in this paper was partly supported by: Portuguese Foundation for Science and Technology within project FCT/UTA-Est/MAI/0010/2009; the North Portugal Regional Operational Program (ON.2 - O Novo Norte), under the National Strategic Reference Framework (NSRF), through the European Regional Development Fund (ERDF)

\section{REFERENCES}

[1] F. Xia, N.Y. Asabere, A.M. Ahmed,J. Li, X. Kong "Mobile Multimedia Recommendation in Smart Communities: A Survey" Access, IEEE, vol.1, no., pp.606,624, 2013.
[2] F. Ricci, "Mobile recommender systems," Information Technology \& Tourism, vol. 12, no. 3,Pp. 205-231,2010.

[3] Z. Yu, X. Zhou, D. Zhang, C-Y Chin, X. Wang, J. Men “ Supporting Context-Aware Media Recommendations for Smart Phones". IEEE Pervasive Computing, Vol.5, No.3, pp. 68-75, (2006).

[4] G. Adomavicius et..al., "Incorporating contextual information in recommender system using a multidimensional approach", ACM Transactions on Information Systems, Vol. 23, No. 1, 2005, pp. 103145.

[5] A. B. Benitez, D. Zhong, S.F. Chang, J.R. Smith, " MPEG-7 MDS Content Description Tools and Applications" W. Skarbek (Ed.): Computer Analysis of Images and Patterns, Lecture Notes in Computer Science 2124, Springer, (2001).

[6] I. Burnett, F. Perreira, R. Vande Walle and R.Koene, "The MPEG-21 Book”. John Wiley and Sons Ltd,, (2006)

[7] B. Mobasher. "Contextual user modeling for Recommendation". Keynote at the 2nd Workshop on Context-Aware Recommender Systems, 2010.

[8] O.D.Lara and M.A. Labrador. " A Survey on Human Activity Recognition using Wearable Sensors". Communications Surveys \& Tutorials, IEEE, vol.15, no.3, pp.1192,1209, Third Quarter 2013, doi: 10.1109/SURV.2012.110112.00192.

[9] G. Adomavicius, B. Mobasher, F. Ricci, A. Tuzhilin "ContextAware Recommender Systems". AI Magazine. 32(3),2011, 67-80.

[10] A. Aamodt, E. Plaza. ."Case-Based Reasoning: Foundational Issues, Methodological Variations, and System Approaches", in AI Communications 7, no. 1, (1994)

[11] M.T. Andrade, S. Dogan, A. Carreras, V. Barbosa, H.K. Arachchi, J. Delgado, A. M.Kondoz. "Advanced Delivery of Sensitive Multimedia Content for better serving User Expectations in Virtual Collaboration Applications". Multimedia Tools Applications. 58(3): pp. 633-661, 2012.

[12] X. Wang, D. Rosenblum Y. Wang. "Context-aware mobile music recommendation for daily activities". Proceedings of the 20th ACM international conference on Multimedia, October 29-November 02, 2012, Nara, Japan.

[13] CV. Ostuni et.al. "Mobile Movie Recommendation with Linked Data". LNCS Vol.8127, pp.400-415, 2013.

[14] T.D. Pessemier, S. Dooms, L. Martens. "Context-aware Recommendation through context and activity recognition in a mobile environment". Multimed Tools Appl. doi: 10.1007/s11042-013-1582$\mathrm{x}(2013)$.

[15] J. Kwapisz, G. Weiss, S. Moore. "Activity Recognition using Cell Phone Accelerometers". ACM SIGKDD Explorations Newsletter, 12 (2), 2010, 74-82

[16] T. Hussein, T.. Linder,W. Gaulke, and J. Ziegler. "A framework and an architecture for context-aware group recommendations". In Gwendolyn L. Kolfschoten, Thomas Herrmann, and Stephan Lukosch, editors, CRIWG, volume 6257 of Lecture Notes in Computer Science, pages 121-128. Springer, 2010.

[17] P. Resnick, N. Iacovou, M. Suchak, P. Bergstrom, and J. Riedl,"Grouplens: An open architecture for collaborative filtering of netnews", In Proceedings of ACM CSCW'94 Conference on Computer Supported Cooperative Work, Sharing Information and Creating Meaning, pages, 1994, 175-186.

[18] Java EE Services.http://docs.oracle.com/javaee/6/tutorial/doc/gijqy.html[Acce ssed in October,2013]

[19] O. Bouidghaghen, L. Tamine-Lechani, and M. Boughanem. "Dynamically personalizing search results for mobile users". In Proc. of Flexible Query Answering Systems, pages 293-298, 2009.

[20] X.H. Wang., D.Q. Zhang, T. Gu, and H.K.Punk." Ontology Based Context Modeling and Reasoning using OWL". In Workshop Proceedings ofthe 2nd IEEE Conference on Pervasive Computing and Communications (PerCom2004) (Orlando, FL,USA, March 2004), pp. 18-22.

[21] G. Adomavicius and A. Tuzhilin "Towards the next Generation of Recommender Systems: A survey of the State-of-the-art and Possible Extensions". IEEE Transactions on Knowledge and Data Engineering, 17 (6), 200, 734-749

[22] A.M. Otebolaku and M.T. Andrade. "Recognizing High-Level Contexts from Smartphone Built-In Sensors for Mobile Media Content Recommendation". Mobile Data Management (MDM), 2013 IEEE 14th International Conference on , vol.2, no., pp.142,147, 3-6 June 2013.

[23] MULTICAO: http://telecom.inescporto.pt/owl/multicao/ 